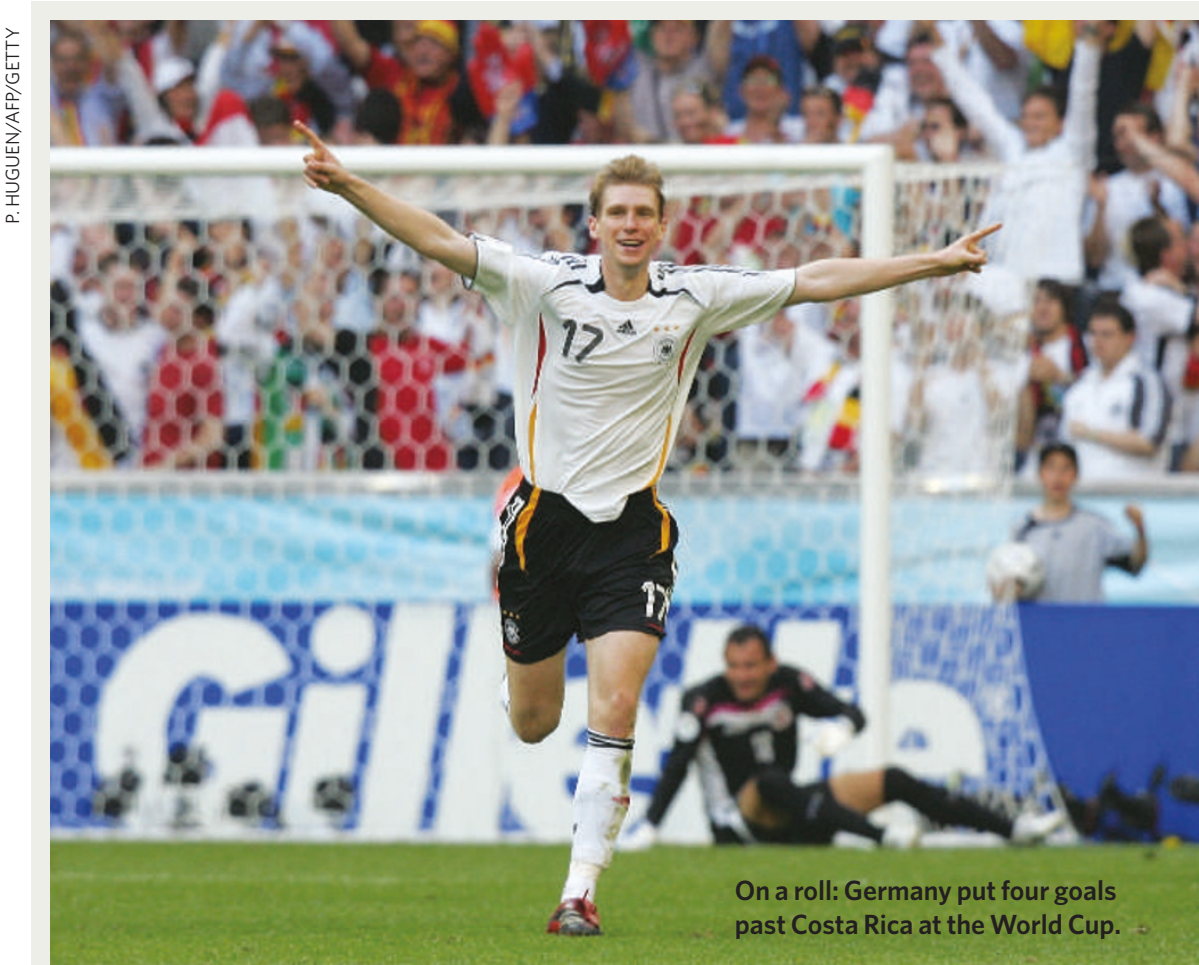

\title{
Goal fever at the World Cup
}

As the World Cup kicks off in Germany, mathematicians say they have proved one of soccer's classic clichés - the idea that when a team scores, the resulting confidence boost helps players romp to victory with a flurry of goals.

Although often seen as wishful thinking rather than football fact, such goal fever can be seen in football results stretching back decades, says mathematician Martin Weigel of Heriot-Watt University in Edinburgh, UK.

Much research has been geared towards improving tactics and understanding fan behaviour, but little has been done on factors affecting the distribution of final scores. Small studies carried out since the 1950s have generally assumed that a team has a set chance of scoring in a game this depends on various factors and remains roughly constant throughout a match. This produces a 'normal' distribution of final scores, like a symmetrical bell-curve. But when Weigel and his colleagues at Germany's University of Leipzig examined results from the German men's and women's league (about 22,000 games) and from the finals of all previous World Cup tournaments (3,400 matches), they found that the scores didn't fit such a distribution: high-scoring games happened far too often.

This suggests that teams don't simply score a number of goals proportional to their skill, but rather are spurred on to greater heights after they score. The researchers designed a model in which a team's chances of scoring are multiplied for every goal, and found that it fitted the skewed distribution of final scores perfectly (E. Bittner, A. Nussbaumer, W. Janke \& M. Weigel; preprint available at http://arxiv.org/abs/physics/ 0606016).

The effect is more noticeable in lower-standard leagues than in the World Cup finals, probably because in higher-quality games teams are more evenly matched and so less likely to gain a psychological upper hand. But the boost gained from scoring is likely to be stronger in football, where goals are relatively rare, than in rugby or basketball, say, where both teams would expect to score reasonably often.

Similar effects can be found in other fields, however.

Statisticians refer to situations where the outcome of one experiment influences the outcome of following ones as 'contagious distributions'. This has been seen with accidents, for example, where one mishap can make someone feel insecure and more likely to suffer further calamities.

\section{Michael Hopkin}

\section{For more on the science of} soccer, from goals to gambling, visitnews@nature.com's interactive World Cup special at www.nature.com/news. 